\title{
Factors Associated with Unsuccessful Treatment of Tuberculosis in Arsi-Robe Hospital, Arsi Zone, Ethiopia: A Retrospective Study
}

Addisu Assefa ( $\nabla$ assefaaddisu@gmail.com )

Madda Walabu University https://orcid.org/0000-0002-7700-6297

Ararsa Girma

Madda Walabu University

Helmut Kloos

University of California San Francisco

\section{Research}

Keywords: Tuberculosis, Treatment outcomes, Multivariable logistic regression, Adjusted odds ratio, unsuccessful treatment outcome, Arsi-Robe Ethiopia

Posted Date: January 21st, 2021

DOI: https://doi.org/10.21203/rs.3.rs-151177/v1

License: (c) (i) This work is licensed under a Creative Commons Attribution 4.0 International License. Read Full License 


\section{Abstract}

Background: Tuberculosis remains a major global health problem and ranks along with the human immunodeficiency virus (HIV) as a leading cause of mortality worldwide. The aim of this study was to investigate the treatment outcome of tuberculosis, and factors associated with treatment outcome of tuberculosis in TB patients enrolled in Arsi-Robe Hospital, Oromia regional state, South eastern Ethiopia between January 2013 to December 2017.

Methods: An Institutional-based retrospective study was conducted in Arsi-Robe Hospital from 2013 to 2017 in study patients who had all forms of TB in DOTS clinic. The predictors of treatment outcomes were analyzed through bivariate and multivariable logistic regression analysis and a $P$-value $<0.05$ were considered statistically significant.

Results: Out of the 257 registered TB patients, most of them were males (57.9\%), from rural areas (62.6\%) and in age of 15-24 category (39.3\%). PTB-, PTB+ and EPTB were recorded in $48.2 \%, 32 \%$ and $19.8 \%$ of the patients, respectively. Among all cases, $8.6 \%$ had TB-HIV co-infection. Among all TB cases, $84.0 \%$ had successful treatment outcome. TB patients from urban areas (AOR: $3.34,95 \% \mathrm{Cl}: 1.338 .38, P=0.01$ ), with failure treatment (AOR: $6.66,95 \% \mathrm{Cl}=1.12-39.57 ; P=0.037$ ) and HIV positive (AOR: $4.92,95 \% \mathrm{Cl}=1.38-17.51 ; P=0.014)$ had higher odd of unsuccessful treatment outcome of tuberculosis. However, TB patients with PTB+ (AOR: $0.1470,95 \% \mathrm{Cl}=0.031-0.687 ; P=0.015)$ and EPTB (AOR: $0.194,95 \% \mathrm{Cl}=0.054-0.688 ; P=0.011)$ had significantly lesser odd of unsuccessful treatment outcome.

Conclusions: Being urban resident, treatment failure and HIV positive considerably challenge the treatment outcome of tuberculosis, but being PTB+ and EPTB were associated with higher treatment success rate of TB. Continuous follow-up of patients with unsuccessful treatment outcome of tuberculosis with strengthened implementation of the DOTs strategies are suggested.

Trial Registered: retrospectively registered

\section{Background}

Tuberculosis (TB) is one of the top 10 causes of death, and the leading cause from a single infectious agent (above HIV/AIDS); millions of people continue to fall sick with the disease worldwide each year [1]. According to WHO [1], an estimated 1.3 million (range, 1.2-1.4 million) deaths from TB among HIV-negative people in 2017 and an additional 300, 000 (range, 266 000-335 000) deaths from TB among HIV-positive people were reported. One-third of the world population was infected by Mycobacterium but only $10 \%$ were predicted to be sick with active TB [2].

The African Region had $25 \%$ of the world's TB cases, but the most severe burden relative to population (237 incident cases per 100000 population on average, as compared to global average of 133) and the highest rates of cases and deaths per capital [1]. Ninety-five percent of all TB cases and $99 \%$ of deaths occur in developing countries, with the greatest burden in sub-Saharan Africa and South East Asia [3]. In sub Saharan Africa, it was estimated that a single TB patient with active disease if not treated can infect on average 10-15 people every year [4]. According to Global TB report 2018 [1], the prevalence of TB in Ethiopia was $164 / 100,000$ in 2017 , leading to an annual mortality rate of 27.5 per 100,000 populations in 2017 . TB affects all countries and all age groups, but overall the best estimated for 2017 were that $90 \%$ of cases were adults (aged $\geq 15$ years), $64 \%$ were male, $9 \%$ were people living with HIV ( $72 \%$ of them in Africa) and two thirds were in eight countries: India, China, Indonesia, the Philippines, Pakistan, Nigeria, Bangladesh and South Africa [1].

The risk factors of TB infection have been revealed from different studies. Human immunodeficiency virus (HIV), smoking, diabetes mellitus (DM), alcohol use and under-nutrition, use of immunosuppressive drugs and being of young age, socioeconomic and behavioral aspects have been found in increasing the susceptibility to TB infection [4-6]. In Ethiopia, the reported high mortality rate among TB patients has been ascribed to co-infection with HIV and the emergence of MDR M. tuberculosis strains [7]. Retrospective studies conducted in Bale Robe Hospital [8], Gondar [9], Arsi Asella Hospital [10] showed that human immunodeficiency virus as a determinant of tuberculosis treatment outcome.

Loading [MathJax]/jax/output/CommonHTML/fonts/TeX/fontdata.js 
A DOTs (Directly Observed Treatment Short Course) is a global strategy launched by WHO in 1994 for prevention and control Tuberculosis. The DOTs program principally employs a direct observation of treatment means where health professionals must watch the patient taking each dose to ensure correct treatment and notice any problem rapidly to take action [11]. Between 1995 and 2012, 56 million people were successfully treated for TB in countries that had adopted the DOTS/Stop TB Strategy which saved approximately 22 million lives [3].

In Ethiopia, the DOTs strategy was started in Arsi Zone and Bale zones in 1992. It has been subsequently scaled up and implemented at national level [7], and almost all public, private and non-governmental health facilities implement the strategy [12], but the case detection and treatment outcome vary across different regions of Ethiopia [13]. Retrospective cohort studies of treatment outcome all forms of conducted in Asella Teaching Hospital during 2004 to 2014 revealed a TSR of 82.9\% [10]. Studies of treatment success rate of smear positive pulmonary TB of 25 districts of Arsi zone, central Ethiopia from September 2004 to 2011 revealed a treatment success rate that ranged from 69.3 to $92.5 \%$, but, the TSR in Arsi-Robe district (82.4\%) [14] was still below the WHO TSR.

Factors contributing to treatment outcome of TB are likely to vary depending on the local settings of populations. Being older age, living in rural areas, sputum smear negative treatment category at initiation of treatment, smear positive sputum test result at second month after initiation treatment, retreatment cases and HIV positive status were reported as predictors factors associated with unsuccessful TB treatment outcome [10,13-14]. It was suggested that analysis of factors affecting treatment outcomes may help to improve performance of DOTS services and provide useful evidence for decision making in disease control programs [15]. However, no study has assessed the treatment outcome of TB in Arsi-Robe hospital, South eastern Ethiopia from January 2013 to December 2017. To the best of knowledge, this is the first and most comprehensive study that specifically addressed the treatment outcome of TB and associated risk factors under DOTS strategy in this public hospital.

\section{Methods}

\section{Study Design and Setting}

The study was conducted Arsi-Robe Hospital, located in Arsi-Robe town in Arsi Administrative Zone, Southeastern Ethiopia. The town is situated at a longitude, a latitude and elevation of $09^{\circ} 36^{\prime} \mathrm{N}, 39^{\circ} 08^{\prime} \mathrm{E}$, and 2435 meters above sea level, respectively. Arsi-Robe town is located at $226 \mathrm{Km}$ east of Addis Ababa and $101 \mathrm{Km}$ from Arsi zonal town, Asella. The hospital has commenced its clinical services (including TB treatment in DOTs clinic) since March 2011. In the hospital, the DOTS clinic is operating under the National Tuberculosis and Leprosy Control Program (NTLCP) of Ethiopia, under which the diagnosis different types of TB was conducted using detection of acid fast bacilli in (AFB) under microscopy. Patients diagnosed with tuberculosis were treated using combination of four drugs (rifampicin, isoniazid, pyrazinamide and ethambutol (2RHZE) according to the Ethiopian national tuberculosis and leprosy control guideline (NTLCP guideline) [12]. The Hospital is serving more than half a million population of the town and the adjacent districts.

An institutional retrospective design was used to study the treatment outcomes of TB and associated risks in tuberculosis patients registered from January 2013 to December 2017 at Arsi-Robe Hospital located at Arsi-Robe town. All forms of TB cases diagnosed with smear, and/or radiography for all forms of TB which were registered in the TB clinic of the Hospital and who took anti-TB treatment except MDR-TB between January 2013 to December 2017. Registries in which treatment outcomes were missing and patients who transferred to other districts were excluded from the treatment outcome evaluation, as information on their treatment outcome was not available. Those cases that were under ongoing TB treatment during data recording by the researcher were also excluded. Variables such as demographic data (patient's age, sex, and residence), clinical history (type of TB, patient category during the start of treatment), and information including HIV status of the patient, year of treatment and treatment outcomes were recovered from patients' registration document using structured questionnaire by the trained nurse working in the Arsi-Robe hospital. The completed datasets were supervised by the principal investigator for completeness and consistency.

\section{Case and Treatment Outcome Definitions}

Loading [MathJax]/jax/output/CommonHTML/fonts/TeX/fontdata.js 
Case definition and treatment outcome were defined based on the standard definitions of the National Tuberculosis and leprosy control program (NTLCP) guideline of Ethiopia for the diagnosis and treatment of TB case [12].

\section{Cases definitions}

- New case. A patient who never had treatment for TB, or has been on anti-TB treatment for less than four weeks in the past.

- Relapse. A patient who declared cured or treatment completed of any form of TB in the past, but who reports back to the health service and is now found to be AFB smear-positive or culture positive.

- Treatment failure. A patient who, while on treatment, is smear-positive at the end of the fifth month or later, after commencing.

- Default. A patient previously recorded as defaulted from treatment and returns to the health facility with smear-positive sputum.

- Transfer-in. A patient who is transferred-in to continue treatment in a given treatment unit after starting treatment in another treatment unit for at least four weeks;

- Others. A patient who does not fit in any of the above mentioned categories.

\section{Treatment outcomes definitions}

- Cured. A patient whose sputum smear or culture was positive at the beginning of the treatment but who was smear- or culture-negative in the last month of treatment and on at least one previous occasion.

- Treatment completed. A patient who completed treatment but who does not have a negative sputum smear or culture result in the last month of treatment and on at least one previous occasion.

- Treatment failure. A patient whose sputum smear or culture is positive at 5 months or later during treatment or patients found to harbor a multidrug-resistant (MDR) strain at any point of time during the treatment, whether they are smearnegative or - positive.

- Defaulter. A patient who has been on treatment for at least four weeks and whose treatment was interrupted for eight or more consecutive weeks.

- Died. A patient died from any cause during the course of treatment.

- Transferred out. A patient who started treatment and has been transferred to another reporting unit and for whom the treatment outcome is not known at the time of evaluation of treatment results.

- Successful treatment outcome. If TB patients were cured or treatment completed with resolution of symptoms.

- Unsuccessful treatment outcome. If treatment of TB patients resulted in treatment failure, default, or death.

Treatment success rate (TSR). The sum of the percentages of cured and patients who completed treatment.

\section{Data Analysis}

Data were entered and analyzed using SPSS version 21 statistical software. Descriptive statistics were computed and employed to summarize socio-demographic data, clinical data and treatment outcome. Pearson Chi-square test was used to evaluate the association of independent factors to tuberculosis types and the difference was significant at $P<0.05$. Bivariate and multivariable logistic regression was used to explore possible associations between dependent variable (treatment outcome) and independent variables. The independent variables were checked for the presence multicollinearity using Variance Inflation Factor (VIF). The goodness of fit of the employed model was evaluated using the Hosmer-Lemeshow test. The COR of independent variables whose $95 \%$ confidence interval $(\mathrm{Cl})$ excluding one were fitted to multivariable logistic regression to calculate the adjusted odd ratio (AOR). In AOR, the 95\% confidence interval (Cl) of odds ratio (OR) excluding one was significantly associated with the corresponding dependent variable.

\section{Results}

\section{Patients' characteristics}


A total of 257 TB patients were registered for DOTs program at Arsi-Robe Hospital, Oromia, between January 2013 and December 2017. Majority of them were males (57.9\%) and rural residents $(62.6 \%)$. The patients' age ranged from 1-80 years and had mean age \pm SD of $27.63 \pm 14.13$ years. Most of them (74.4\%) were in the economically productive age group (1544 years) and in the weight category of $45-54$ years (38.1\%). Among TB patients, the majority of the cases were new cases (69.6\%) and HIV negative (91.4\%) (Table 1). 
Table 1

Frequency and percentage of TB patients at Arsi-Robe Hospital, Ethiopia

\begin{tabular}{|c|c|c|c|}
\hline Variable & Categories & Total TB patients $(\mathrm{N}=257)$ & Percentage \\
\hline \multirow[t]{2}{*}{ Sex } & Female & 108 & 42.1 \\
\hline & Male & 149 & 57.9 \\
\hline \multirow[t]{2}{*}{ Residence } & Rural & 161 & 62.6 \\
\hline & Urban & 96 & 37.4 \\
\hline \multirow[t]{7}{*}{ Age Groups } & $<=14$ & 29 & 11.3 \\
\hline & $15-24$ & 101 & 39.3 \\
\hline & $25-34$ & 58 & 22.6 \\
\hline & $35-44$ & 32 & 12.5 \\
\hline & $45-54$ & 18 & 7.0 \\
\hline & $55-64$ & 14 & 5.4 \\
\hline & $65+$ & 5 & 1.9 \\
\hline \multirow[t]{6}{*}{ TB Patient treatment Category } & New & 179 & 69.6 \\
\hline & Relapse & 13 & 5.1 \\
\hline & Default & 34 & 13.2 \\
\hline & Failure & 16 & 6.2 \\
\hline & Transfer in & 8 & 3.1 \\
\hline & Other & 7 & 2.7 \\
\hline \multirow[t]{7}{*}{ Weight(Kg) } & $<=14$ & 13 & 5.1 \\
\hline & $15-24$ & 14 & 5.4 \\
\hline & $25-34$ & 14 & 5.4 \\
\hline & $35-44$ & 62 & 24.1 \\
\hline & $45-54$ & 98 & 38.1 \\
\hline & $55-64$ & 49 & 19.1 \\
\hline & $65+$ & 7 & 2.7 \\
\hline \multirow[t]{2}{*}{ HIV Status } & Negative & 235 & 91.4 \\
\hline & Positive & 22 & 8.6 \\
\hline \multirow[t]{3}{*}{ Types of TB } & PTB- & 124 & 48.3 \\
\hline & PTB+ & 82 & 31.9 \\
\hline & ЕРТВ & 51 & 19.8 \\
\hline \multirow[t]{4}{*}{ Year of Treatment } & 2013 & 64 & 24.9 \\
\hline & 2014 & 53 & 20.6 \\
\hline & 2015 & 48 & 18.7 \\
\hline & 2016 & 44 & 17.1 \\
\hline
\end{tabular}




\begin{tabular}{|lllll|}
\hline Variable & Categories & Total TB patients $(\mathbf{N}=257)$ & Percentage \\
\hline & 2017 & 48 & 18.7 \\
\hline
\end{tabular}

\section{Patients' characteristics and type of TB}

Based on TB types, $48.2 \%, 32.0 \%$ and $19.8 \%$ of the patients were diagnosed for PTB-, PTB+, and EPTB, respectively (Tables 2 ). The percentage of patients diagnosed for different types of TB did not significantly vary between sex, residence, age categories, weight and year of the treatment of the patients $(P>0.05)$. Male TB patients had slightly higher frequency of both PTB- (49.7\%) and PTB+ $(32.9 \%)$ than female patients, but EPTB was higher in female $(23.1 \%)$ than males $(17.4 \%)$. The percentage of patients who diagnosed for different TB types significantly were varied with HIV status of the TB patients $\left(\chi^{2}=6.35 ; P=0.043\right)$. HIV positive TB patients had significantly higher PTB- (54.5\%) followed by PTB+ (45.5\%) as compared to HIV negative TB patients. The frequency of PTB-, PTB + and EPTB were higher in 2015 (62.5\%), 2017 (43.6\%) and in 2016 (27.2\%), respectively as compared to other years (Table 2). 
Table 2

Patients' characteristics and type of TB at Arsi-Robe Hospital, 2013-2017

\begin{tabular}{|c|c|c|c|c|c|c|c|}
\hline \multirow[t]{2}{*}{ Variables } & \multirow[t]{2}{*}{ Categories } & \multicolumn{3}{|c|}{ Types of Tuberculosis } & \multirow{2}{*}{$\begin{array}{l}\text { Total } \\
\mathbf{N}(\%)\end{array}$} & \multirow[t]{2}{*}{$x^{2}$} & \multirow[t]{2}{*}{ P-value } \\
\hline & & PTB- (\%) & PTB+(\%) & EPTB (\%) & & & \\
\hline \multirow[t]{2}{*}{ Sex } & Female & $50(46.3)$ & $33(30.6)$ & $25(23.1)$ & $108(42.1)$ & \multirow[t]{2}{*}{1.28} & \multirow[t]{2}{*}{0.516} \\
\hline & Male & $74(49.7)$ & $49(32.9)$ & $26(17.4)$ & $149(57.9)$ & & \\
\hline \multirow[t]{2}{*}{ Residence } & Rural & $72(44.7)$ & $54(33.5)$ & $35(21.7)$ & $161(62.6)$ & \multirow[t]{2}{*}{2.25} & \multirow[t]{2}{*}{0.324} \\
\hline & Urban & $52(54.2)$ & $28(29.2)$ & $16(16.7)$ & $96(37.4)$ & & \\
\hline \multirow[t]{7}{*}{ Age groups } & $<=14$ & $17(58.6)$ & $7(24.1)$ & $5(17.2)$ & $29(11.3)$ & \multirow[t]{7}{*}{18.61} & \multirow[t]{7}{*}{0.098} \\
\hline & $15-24$ & $36(35.6)$ & $38(37.6)$ & $27(26.7)$ & $101(39.3)$ & & \\
\hline & $25-34$ & $27(46.6)$ & $18(31.0)$ & $13(22.4)$ & $58(22.6)$ & & \\
\hline & $35-44$ & $21(65.6)$ & $8(25.0)$ & $3(9.4)$ & $32(12.5)$ & & \\
\hline & $45-54$ & $13(72.2)$ & $4(22.2)$ & $1(5.6)$ & $18(7.0)$ & & \\
\hline & $55-64$ & $7(50.0)$ & $5(35.7)$ & $2(14.3)$ & $14(5.4)$ & & \\
\hline & $65+$ & $3(60.0)$ & $2(40.0)$ & $0(0.0)$ & $5(1.9)$ & & \\
\hline \multirow[t]{7}{*}{ Weight (Kg) } & $<=14$ & $7(53.8)$ & $3(23.1)$ & $3(23.1)$ & $13(5.1)$ & \multirow[t]{7}{*}{11.05} & \multirow[t]{7}{*}{0.531} \\
\hline & $15-24$ & $9(64.3)$ & $2(14.3)$ & $3(21.4)$ & $14(5.4)$ & & \\
\hline & $25-34$ & $10(71.4)$ & $3(21.4)$ & $1(7.1)$ & $14(5.4)$ & & \\
\hline & $35-44$ & $32(51.6)$ & $20(32.2)$ & $10(16.1)$ & $62(24.1)$ & & \\
\hline & $45-54$ & $39(39.8)$ & $37(37.7)$ & $22(22.4)$ & $98(38.1)$ & & \\
\hline & $55-64$ & $22(44.9)$ & $16(32.6)$ & $11(22.4)$ & $49(19.0)$ & & \\
\hline & $65+$ & $5(71.4)$ & $1(14.3)$ & $1(14.3)$ & $7(2.7)$ & & \\
\hline \multirow[t]{6}{*}{ TB Patient treatment Category } & New & $85(47.5)$ & $57(31.8)$ & $37(20.7)$ & $179(69.6)$ & \multirow[t]{6}{*}{10.21} & \multirow[t]{6}{*}{0.422} \\
\hline & Relapse & $5(38.4)$ & $6(46.1)$ & $2(15.4)$ & $13(5.1)$ & & \\
\hline & Defaulted & $16(47.1)$ & $13(38.2)$ & $5(14.7)$ & $34(13.2)$ & & \\
\hline & Failure & $8(50.0)$ & $6(37.5)$ & $2(12.5)$ & $16(6.2)$ & & \\
\hline & Transfer in & $5(62.5)$ & $0(0.0)$ & $3(37.5)$ & $8(3.1)$ & & \\
\hline & Other & $5(71.4)$ & $0(0.0)$ & $2(28.6)$ & $7(2.7)$ & & \\
\hline \multirow[t]{2}{*}{ HIV Status } & Negative & $112(47.7)$ & $72(30.6)$ & $51(21.7)$ & $235(91.4)$ & \multirow[t]{2}{*}{6.35} & \multirow[t]{2}{*}{0.043} \\
\hline & Positive & $12(54.5)$ & $10(45.5)$ & $0(0.0)$ & $22(8.6)$ & & \\
\hline \multirow[t]{5}{*}{ Year of Treatment } & 2013 & $32(50.0)$ & $19(29.7)$ & $13(20.3)$ & $64(24.9)$ & \multirow[t]{5}{*}{12.2} & \multirow[t]{5}{*}{0.054} \\
\hline & 2014 & $25(47.2)$ & $18(33.9)$ & $10(1.9)$ & $53(20.6)$ & & \\
\hline & 2015 & $30(62.5)$ & $8(16.7)$ & $10(20.8)$ & $48(18.7)$ & & \\
\hline & 2016 & $16(36.4)$ & $16(36.4)$ & $12(27.2)$ & $44(17.1)$ & & \\
\hline & 2017 & $21(43.7)$ & $21(43.6)$ & $6(12.5)$ & $48(18.7)$ & & \\
\hline
\end{tabular}




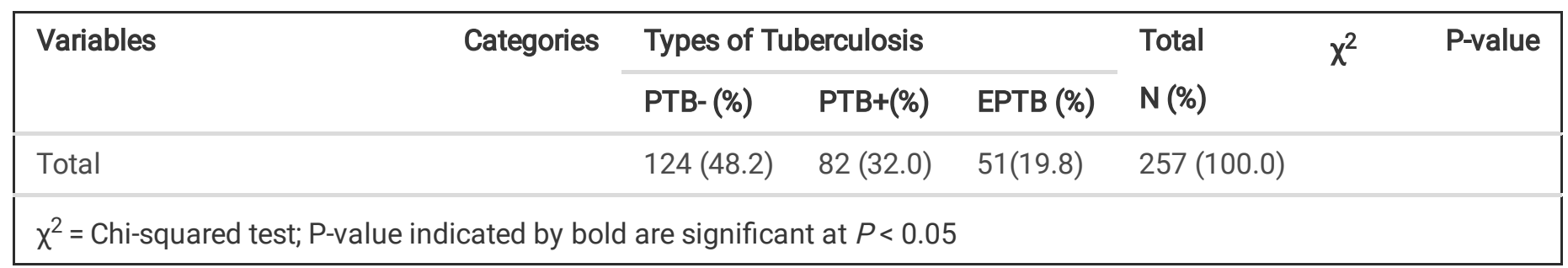

\section{Treatment Outcome}

The treatment outcomes of TB patients who were registered on DOTs program at the Arsi-Robe Hospital, Ethiopia during 2013 to 2017 was indicated in Table 3. Among 257 TB patients who initiated anti-TB treatment, 31.5\% were cured from their TB diseases (Table 3). The highest percentage of cured TB patients was recorded from rural area (34.8\%), in age group of 55-64 years (42.9\%), in relapsed patients (38.5\%), in weight category of 45-54 (37.8\%), in HIV positive (36.4\%), in PTB+ (93.9\%) and during $2017(45.8 \%)$ as compared to the corresponding counterparts. Among TB patients, $52.5 \%$ of the TB patients were completed their treatment. Male TB patients (56.4\%), in the age $\leq 14$ years (72.4\%), transferred in patients (62.5\%), EPTB patients (84.3\%), HIV negative (55.3\%) and TB patients treated during 2015 (64.6\%) had relatively higher rate of treatment completion. 
Table 3

Treatment outcome of TB patients at Arsi-Robe Hospital, Ethiopia, from 2013-2017

\begin{tabular}{|c|c|c|c|c|c|c|}
\hline \multirow[t]{3}{*}{ Variables } & \multirow[t]{3}{*}{ Categories } & \multicolumn{5}{|c|}{ Treatment outcomes } \\
\hline & & Cured & Completed & Defaulted (\%) & Died (\%) & Failure (\%) \\
\hline & & (\%) & (\%) & & & \\
\hline \multirow[t]{2}{*}{ Sex } & Female & $34(31.5)$ & $51(47.2)$ & $10(9.2)$ & $8(7.4)$ & $5(4.6)$ \\
\hline & Male & $47(31.5)$ & $84(56.4)$ & $3(2.0)$ & $7(4.7)$ & $8(5.4)$ \\
\hline \multirow[t]{2}{*}{ Residence } & Rural & $56(34.8)$ & $85(52.8)$ & $9(5.6)$ & $6(3.7)$ & $5(3.1)$ \\
\hline & Urban & $25(26.0)$ & $50(52.1)$ & $4(4.2)$ & $9(9.4)$ & $8(8.3)$ \\
\hline \multirow[t]{7}{*}{ Age groups } & $<=14$ & $7(24.1)$ & $21(72.4)$ & $0(0.0)$ & $1(3.5)$ & $0(0.0)$ \\
\hline & $15-24$ & 37 (36.6) & $53(52.5)$ & $3(3.0)$ & $3(3.0)$ & $5(4.9)$ \\
\hline & $25-34$ & $18(31.0)$ & $30(51.7)$ & $4(6.9)$ & $3(5.2)$ & $3(5.2)$ \\
\hline & $35-44$ & $7(21.9)$ & $16(50.0)$ & $4(12.5)$ & $2(6.3)$ & $3(9.3)$ \\
\hline & $45-54$ & $4(22.2)$ & $7(38.9)$ & $2(11.1)$ & $4(22.2)$ & $1(5.6)$ \\
\hline & $55-64$ & $6(42.9)$ & $6(42.9)$ & $0(0.0)$ & $1(7.1)$ & $1(7.1)$ \\
\hline & $65+$ & $2(40.0)$ & $2(40.0)$ & $0(0.0)$ & $1(20.0)$ & $0(0.0)$ \\
\hline \multirow[t]{6}{*}{ TB patient treatment category } & New & $58(32.2)$ & $103(57.5)$ & $4(2.2)$ & $4(2.2)$ & $10(5.6)$ \\
\hline & Relapse & $5(38.5)$ & $6(46.2)$ & $0(0.0)$ & $2(15.4)$ & $0(0.0)$ \\
\hline & Defaulted & $12(35.3)$ & $12(35.5)$ & $4(11.7)$ & $5(14.7)$ & $1(2.9)$ \\
\hline & Failure & $5(31.3)$ & $6(37.5)$ & $3(18.8)$ & $2(12.5)$ & $0(0.0)$ \\
\hline & Transfer in & $0(0.0)$ & $5(62.5)$ & $2(25.0)$ & $0(0.0)$ & $1(12.5)$ \\
\hline & Other & $1(14.3)$ & $3(42.9)$ & $0(0.0)$ & $2(28.5)$ & $1(14.3)$ \\
\hline \multirow[t]{7}{*}{ Weight (Kg) } & $<=14$ & $3(23.1)$ & $9(69.2)$ & $0(0.0)$ & $1(7.7)$ & $0(0.0)$ \\
\hline & $15-24$ & $2(14.3)$ & $10(71.4)$ & $0(0.0)$ & $1(7.1)$ & $1(7.1)$ \\
\hline & $25-34$ & $5(35.7)$ & $6(42.9)$ & $0(0.0)$ & $2(14.3)$ & $1(7.1)$ \\
\hline & $35-44$ & $19(30.6)$ & $27(43.5)$ & $5(8.1)$ & $6(9.7)$ & $5(8.1)$ \\
\hline & $45-54$ & $37(37.8)$ & $52(53.1)$ & $5(5.1)$ & $2(2.0)$ & $2(2.0)$ \\
\hline & $55-64$ & $14(29.2)$ & $28(58.3)$ & $1(2.1)$ & $3(6.3)$ & $3(6.3)$ \\
\hline & $65+$ & $1(14.3)$ & $3(42.8)$ & $2(28.6)$ & $0(0.0)$ & $1(14.3)$ \\
\hline \multirow[t]{2}{*}{ HIV status } & Negative & $73(31.1)$ & $130(55.3)$ & $11(4.7)$ & $11(4.7)$ & $10(4.3)$ \\
\hline & Positive & $8(36.4)$ & $5(22.7)$ & $2(9.1)$ & $4(18.2)$ & $3(13.6)$ \\
\hline \multirow[t]{3}{*}{ Type of TB } & PTB- & $4(3.2)$ & $91(73.4)$ & $2(9.1)$ & $4(18.2)$ & $3(13.6)$ \\
\hline & PTB+ & 77 (93.9) & $1(1.2)$ & $0(0.0)$ & $3(3.7)$ & $1(1.2)$ \\
\hline & ЕРТВ & $0(0.0)$ & $43(84.3)$ & $3(5.9)$ & $1(2.0)$ & $4(7.8)$ \\
\hline \multirow[t]{2}{*}{ Year of treatment } & 2013 & $19(29.7)$ & $30(46.9)$ & $6(9.4)$ & $4(6.3)$ & $5(7.8)$ \\
\hline & 2014 & $14(26.4)$ & $32(60.4)$ & $0(0.0)$ & $3(5.7)$ & $4(7.5)$ \\
\hline
\end{tabular}

Page $10 / 19$ 


\begin{tabular}{|lllllll|}
\hline Variables & Categories & \multicolumn{2}{l}{ Treatment outcomes } \\
& & $\begin{array}{l}\text { Cured } \\
\text { (\%) }\end{array}$ & Completed & Defaulted (\%) & Died (\%) & Failure (\%) \\
& & $(\%)$ & & & \\
\hline & 2015 & $8(16.7)$ & $31(64.6)$ & $4(8.3)$ & $4(8.3)$ & $1(2.1)$ \\
\hline 2016 & $18(40.9)$ & $22(50.0)$ & $1(2.3)$ & $1(2.3)$ & $2(4.5)$ \\
\hline 2017 & $22(45.8)$ & $20(41.7)$ & $2(4.2)$ & $3(6.2)$ & $1(2.1)$ \\
\hline Total & $81(31.5)$ & $135(52.5)$ & $13(5.1)$ & $15(5.8)$ & $13(5.1)$ \\
\hline
\end{tabular}

However, $5.1 \%$ of the TB patients were default their TB treatment. The frequency of defaulted TB patients were high in female TB patients (9.2\%), in age of 35-44 years (12.5\%), in transferred in cases $(25 \%)$, in weight of $65+(28.6 \%)$, in HIV positive patients (9.1\%), in PTB- patients (9.1\%), and in patients treated during 2015 (8.3\%) as compared to other counterparts. Our study found that $5.8 \%$ of the TB patients were died. The percentage of died TB was higher in female study subjects (7.4\%), in urban residents (9.4\%), in the age of 45-54 (22.2\%), in HIV positive patients (18.2\%), in PTB- patients (18.2\%) and in TB patients treated during $2015(8.3 \%)$ as compared to other counterparts. The percentage of TB patients with failure treatment was $5.2 \%$. TB patients who were urban residents (8.3\%), in the age of 35-44 years (9.3\%), HIV positive (13.6\%) and PTB- (13.6\%) and treated during 2013 (7.8\%) had relatively high rate of treatment failure compared to other counterparts (Table 3 ).

\section{Treatment success rate (TSR)}

The treatment success rate of TB patients treated during the study period was indicated in Table 4 . Of the total 257 patients who initiated anti-TB treatment, $216(84.0 \%)$ had successfully treated, and $41(16.0 \%)$ had unsuccessful treatment outcome. TB patients who were male (87.9\%), rural residents (87.6\%), and in the age $\leq 14$ years (96.6\%), body weight of $\leq 14 \mathrm{Kg}(92.3 \%)$, defaulters (89.9\%), HIV negative (86.4\%), and treated during 2016 (90.9\%) had high treatment success rate as compared to their counterparts (Table 4). 
Table 4

Factors associated with unsuccessful treatment outcome of TB patients in Arsi-Robe Hospital, Ethiopia

\begin{tabular}{|c|c|c|c|c|c|c|c|}
\hline \multirow[t]{2}{*}{ Variables } & & \multicolumn{6}{|c|}{ Treatment outcome } \\
\hline & & $\begin{array}{l}\text { Successful } \\
(\%)\end{array}$ & $\begin{array}{l}\text { Unsuccessful } \\
\text { (\%) }\end{array}$ & COR $(95 \% \mathrm{Cl})$ & $\begin{array}{l}\mathrm{P}- \\
\text { value }\end{array}$ & AOR (95\% Cl) & $\begin{array}{l}\mathrm{P} \text { - } \\
\text { value }\end{array}$ \\
\hline \multirow[t]{2}{*}{ Sex } & Female & 85 (78.7) & $23(21.3)$ & 1 & - & 1 & - \\
\hline & Male & $131(87.9)$ & $18(12.1)$ & $\begin{array}{l}0.50(0.26- \\
0.99)\end{array}$ & 0.049 & $\begin{array}{l}0.92(0.37- \\
2.30)\end{array}$ & 0.864 \\
\hline \multirow[t]{2}{*}{ Residence } & Rural & $141(87.6)$ & $20(12.4)$ & 1 & - & 1 & - \\
\hline & Urban & $75(78.1)$ & $21(21.9)$ & $\begin{array}{l}1.97(1.01- \\
3.87)\end{array}$ & 0.048 & $\begin{array}{l}3.34(1.33- \\
8.38)\end{array}$ & 0.01 \\
\hline \multirow[t]{7}{*}{ Age groups } & $<=14$ & $28(96.6)$ & $1(3.4)$ & 1 & - & - & - \\
\hline & $15-24$ & $90(89.1)$ & $11(10.9)$ & $\begin{array}{l}7.0(0.36- \\
135.52)\end{array}$ & 0.198 & - & - \\
\hline & $25-34$ & $48(82.7)$ & $10(17.2)$ & $\begin{array}{l}2.04(0.21- \\
19.97)\end{array}$ & 0.538 & - & - \\
\hline & $35-44$ & $23(71.9)$ & $9(28.1)$ & $\begin{array}{l}1.2(.121- \\
11.90)\end{array}$ & 0.876 & - & - \\
\hline & $45-54$ & $11(61.1)$ & $7(38.9)$ & $\begin{array}{l}0.64(0.06- \\
6.52)\end{array}$ & 0.705 & - & - \\
\hline & $55-64$ & $12(85.7)$ & $2(14.3)$ & $\begin{array}{l}0.40(.036- \\
4.27)\end{array}$ & 0.443 & - & - \\
\hline & $65+$ & $4(80.0)$ & $1(20.0)$ & $\begin{array}{l}1.5(0.11- \\
21.31)\end{array}$ & 0.765 & - & - \\
\hline \multirow{6}{*}{$\begin{array}{l}\text { TB patient treatment } \\
\text { category }\end{array}$} & New & $24(70.6)$ & $10(29.4)$ & 1 & - & 1 & - \\
\hline & Relapse & $11(68.8)$ & $5(31.2)$ & $\begin{array}{l}1.44(0.28- \\
7.21)\end{array}$ & 0.657 & $\begin{array}{l}1.24(0.18- \\
8.44)\end{array}$ & 0.827 \\
\hline & Default & $161(89.9)$ & $18(10.1)$ & $\begin{array}{l}1.32(0.22- \\
7.82)\end{array}$ & 0.76 & $\begin{array}{l}0.73(0.075- \\
7.25)\end{array}$ & 0.795 \\
\hline & Failure & $4(57.1)$ & $3(42.9)$ & $\begin{array}{l}5.37(1.18- \\
24.34)\end{array}$ & 0.029 & $\begin{array}{l}6.66(1.12- \\
39.57)\end{array}$ & 0.037 \\
\hline & $\begin{array}{l}\text { Transfer } \\
\text { in }\end{array}$ & $11(84.6)$ & $2(16.4)$ & $\begin{array}{l}0.80(0.10- \\
6.35)\end{array}$ & 0.833 & $\begin{array}{l}2.94(0.22- \\
39.28)\end{array}$ & 0.415 \\
\hline & Other & $5(62.5)$ & $3(37.5)$ & $\begin{array}{l}3.3(0.41- \\
26.37)\end{array}$ & 0.26 & $\begin{array}{l}1.76(0.165- \\
18.90)\end{array}$ & 0.638 \\
\hline \multirow{5}{*}{$\begin{array}{l}\text { Weight } \\
(\mathrm{Kg})\end{array}$} & $<=14$ & $12(92.3)$ & $1(7.7)$ & 1 & - & - & - \\
\hline & $15-24$ & 12 (85.7) & $2(14.3)$ & )$^{9(0.72-113.02}$ & 0.089 & - & - \\
\hline & $25-34$ & $11(78.6)$ & $3(21.4)$ & $\begin{array}{l}4.5(0.54- \\
37.38)\end{array}$ & 0.164 & - & - \\
\hline & $35-44$ & $46(74.2)$ & $16(25.8)$ & $\begin{array}{l}2.75(.38- \\
19.67)\end{array}$ & 0.314 & - & - \\
\hline & $45-54$ & $89(90.8)$ & $9(9.2)$ & $\begin{array}{l}2.15(0.43- \\
10.69)\end{array}$ & 0.347 & - & - \\
\hline
\end{tabular}

Loading [MathJax]/jax/output/CommonHTML/fonts/TeX/fontdata.js fidence interval; $P$-value indicated by bold are significant at $P<0.05$. 


\begin{tabular}{|c|c|c|c|c|c|c|c|}
\hline & $55-64$ & $42(85.7)$ & 7 (14.3) & $\begin{array}{l}7.42(0.47- \\
38.48)\end{array}$ & 0.17 & - & - \\
\hline & $65+$ & $4(57.1)$ & $3(46.9)$ & $\begin{array}{l}4.5(0.82- \\
24.57)\end{array}$ & 0.082 & - & - \\
\hline \multirow[t]{2}{*}{ HIV status } & Negative & 203 (86.4) & $32(13.6)$ & 1 & - & 1 & - \\
\hline & Positive & $13(59.0)$ & $9(41.0)$ & $\begin{array}{l}4.39(1.74- \\
11.11)\end{array}$ & 0.002 & $\begin{array}{l}4.92(1.38- \\
17.51)\end{array}$ & 0.014 \\
\hline \multirow[t]{3}{*}{ Type of TB } & PTB- & 95 (76.6) & $29(23.4)$ & 1 & - & 1 & - \\
\hline & PTB+ & 78 (95.1) & $4(4.9)$ & $\begin{array}{l}0.27(.078- \\
0.968)\end{array}$ & 0.044 & $\begin{array}{l}0.15(0.03- \\
0.69)\end{array}$ & 0.015 \\
\hline & EPTB & 43 (84.3) & $8(15.7)$ & $\begin{array}{l}0.17(0.057- \\
0.498)\end{array}$ & 0.001 & $\begin{array}{l}0.19(0.05- \\
0.69)\end{array}$ & 0.011 \\
\hline \multirow[t]{5}{*}{ Year of treatment } & 2013 & 49 (76.6) & $15(23.4)$ & 1 & - & 1 & - \\
\hline & 2014 & $46(86.8)$ & $7(13.2)$ & $\begin{array}{l}0.47(.166- \\
1.311)\end{array}$ & 0.148 & & \\
\hline & 2015 & 39 (81.3) & 9 (18.7) & $\begin{array}{l}0.94(0.292- \\
3.018)\end{array}$ & 0.916 & & \\
\hline & 2016 & $40(90.9)$ & $4(9.1)$ & $\begin{array}{l}0.62(0.202- \\
1.900)\end{array}$ & 0.402 & & \\
\hline & 2017 & $42(87.5)$ & $6(12.5)$ & $\begin{array}{l}1.43(0.375- \\
5.441)\end{array}$ & 0.601 & & \\
\hline
\end{tabular}

COR: Crude odds ratio, AOR: Adjusted odds ratio; Cl: Confidence interval; $P$-value indicated by bold are significant at $P<0.05$.

\section{Predictive factors for tuberculosis unsuccessful treatment outcome}

In bivariate logistic regression (COR), variables such as age groups, weight of the patients and year of treatment did not have significant association with unsuccessful treatment outcome of tuberculosis TB $(P>0.05)$. However, sex, residence, TB patients' category, HIV status and types of TB were significantly associated with unsuccessful treatment outcome of tuberculosis $(P<$ 0.05). These variables were fitted to multivariable logistic regression model (Adjusted Odd Ratio, AOR). Accordingly, residence of the TB patients, TB patients' category, HIV status of the patients and TB types were significantly associated with unsuccessful treatment outcome of tuberculosis in multivariable logistic regression analysis $(P<0.05)$.

The odd of unsuccessful treatment outcome was significantly higher in TB patients who were urban resident $(A O R: 3.34, \mathrm{Cl}=$ 1.33-8.38, $P=0.01$ ) compared to rural residence. TB patients with failure treatment had significantly higher odd of unsuccessful treatment outcome (AOR: $6.66, \mathrm{Cl}=1.12-39.57, P=0.037$ ) as compared new patients (reference patient category). Being HIV positive TB patients had significantly higher odd of unsuccessful treatment (AOR: $4.92, \mathrm{Cl}=1.38-17.51, P=0.014$ ) than HIV negative patients. TB patients with PTB+ (AOR: $0.15,95 \% \mathrm{Cl}=0.031-0.69 ; P=0.015)$ and EPTB (AOR: $0.19,95 \% \mathrm{Cl}=$ $0.054-0.69 ; P=0.011)$ had a significantly lower unsuccessful treatment outcome or higher success rate than the PTB- patients (Table 4).

\section{Discussions}

This study was performed to analyze treatment outcome and factors associated with unsuccessful treatment outcome of the TB cases treated at DOTS clinic of Arsi-Robe Hospital, Ethiopia between January 2013 and December 2017 (Table 1). Evaluation of the treatment outcome and associated factors for TB patients has the greatest importance in assessing the effectiveness of DOTS program in a country [16].

In this study, the frequency of male TB patients (57.9\%) was higher than female (42.1\%) in agreements with studies in Arsi zone, Loading [MathJax]/jax/output/CommonHTML/fonts/TeX/fontdata.js studies from Southern Ethiopia (61.3\%) [13] reported higher frequency 
of TB patients in males than females. Globally, gender inequalities have been reported in TB case notification, and TB is more common in males than females [18]. It was suggested that higher proportion of males being exposed to the infection as a result of more social contact or engagement in professions associated with a higher risk for tuberculosis [19], or underutilization of the DOTS service by female patients as a result of various socioeconomic and cultural influences [20] or might be due to the biological differences between male and female in vulnerability to development of active disease [21].

In our study, more TB patients were noted from rural areas (62.6\%) than urban areas (37.4\%). A study from southern Ethiopia also revealed higher frequency of TB patients from rural Ethiopia (66\%) than that of urban areas (44\%) [13]. This might be because of better awareness of tuberculosis and health facility in urban areas than rural areas. The frequency of TB infection was low in earlier ages ( $\leq 14$ years) (Table 1$)$. The low infection frequency in lower ages might be due to vaccination of children with vaccine bacille Calmette-Guérin (BCG) in earlier stages. Ethiopia is one of the countries with BCG coverage in the range of $50-89 \%$ in 2018 [22].

The frequency of TB infection was also higher among new patients as compared other TB treatment categories (Table 1). Similar result was also reported in other study in Debre Tabor [23]. This might indicate that disease was widespread in the society, and the retreatment cases in the society may be the sources for subsequent infection new infections. TB cases were predominantly PTB- (48.3\%) followed by PTB+ (32\%) and the least was EPTB (19.8\%) (Table 1). Similarly, earlier studies from Bale Robe Hospital, south eastern Ethiopia (40.7\%) [8] and from Brazil (65.2\%) reported higher frequency of PTB- over PTB+ [24].

According to WHO [3], continuous tracing of TB treatment outcome, mainly in TB/HIV co-infected patients, is essential. The overall TB-HIV co-infection rate at Arsi-Robe Hospital was 8.6\% (Tables 1 and 2). This was lower than studies in northeastern parts of Ethiopia (24.3\%) [25], and Ethiopia as a whole (29.4\%) [26] and Kenya (41.8\%) [27]. Studies indicated that the unavailability of HIV counseling and testing services or refusal of patients to be tested for HIV [16] or low prevalence of HIV in the study population [28] could contribute to lower TB-HIV co-infection. In this study, PTB- cases had higher number of TB/HIV co-infection than PTB + cases (Table 2). This is the fact that TB cases with HIV are less likely to be smear-positive [29] and high in HIV prevalence countries, the number of patients with SNPT is increasing rapidly [30].

The percentage of cured patients in our study (31.5\%) was higher than the rate in the Bale Robe Hospital (19.7\%) [8]. More than half of the study population completed their treatment. It was higher than study in Gonder Teaching Hospital, North Ethiopia (46.4\%) [9]. The Arsi zone was the zone that historically commenced the first DOTs program [7]. Deployment of health extension workers all across the zones of Ethiopia that provide preventive and curative interventions against TB has been suggested for increase in the treatment outcome (cure and treatment completion) of TB [31].

The failure rate in this study (5.1\%) was higher than in Asella Teaching Hospital, Ethiopia (0.2\%) [10]. Treatment failure is a result of MDR-TB development in previously treated TB cases [32]. Treatment failure steadily decreased across the study period although the trend was not consistent through the years. The defaulting rate in our study (5.1\%) was slightly lower than in Bale Robe Hospital (6\%) [8]. The overall death rates of TB patients in this study (5.8\%) was similar to that in Asella Hospital, central Ethiopia (6\%) [10], but lower than in Arsi Zone for smear positive cases (7.4\%) [14]. In our study, higher death rates occurred in TB patients who were female, urban residents, older, HIV- positive, and in PTB- (Table 2). In agreement with our study, previous studies also reported that older ages (44-54 years), smear- negative pulmonary TB [33] and HIV- positive [34] were more likely to die than their counter parts. High death rates have been reported for urban residents and in PTB- [23] in concordance with our study. According to Dangisso et al. [15], the higher proportion of deaths in PTB- cases than PTB + and EPTB cases might be due to diagnosis and treatment delays, as well as HIV infection among PTB- cases. The recorded higher death in TB patients of older ages might be due to increasing comorbidities as well as the general immunological deterioration with age [35-36].

TB patients who were HIV positive experienced more default (9.1\%), death (18.2\%) and treatment failure (13.6\%) than HIV negative TB patients (Table 3). It was in agreement with study in Arsi Asella [10]. Studies indicated that TB/HIV co-infection has significant impact on treatment outcome [37-38]. HIV affected the performance of TB control programmes by increasing the number of TB cases and by compromising the treatment outcome. 
The overall TSR of all forms of TB in Arsi-Robe Hospital in this study was $84.0 \%$ (Table 4). It was nearly similar with studies in Southwest Ethiopia (85.2\%) [39], but lower than the global target (>90\%) [1], WHO target of Ethiopia for the new cases in the years of 2016 (90\%) [1] and 2017 (96\%) [22], and China (95.01\%) [17]. But, it was higher than Asella Teaching hospital (81.7\%) [10], Bale Robe Hospital (78.2\%) [8], and Kenya (82.4\%) [40]. The variation in the TSR with other studies could be due to differences in variation in DOTs performance in various study areas, socio-economic of the patients, geographic setting, sample size, study period and the TB clinic management (Table 4).

Higher treatment success was noted in male TB patients than female (Table 4). Similar findings were also noted from Addis Ababa [33], and South region [13], but different from studies in Bale Robe Hospital [8], and Arsi Asella hospital [10]. The treatment success rate was high in younger TB patients, and decrease in older ages but the trend was not consistent. Studies indicated that TB patients at higher age might be under immune-compromised situations as a result of associated diseases, and general physiological deterioration with age thereby resulted in poor-treatment outcome [35, 39].

In our study, PTB + had the highest treatment success rates (95.1\%) as compared other TB types, and was higher than Arsi Zone (83.6\%) [14]. The TSR in TB/HIV co-infection cases (59\%) in our study was lower than Bale Robe Hospital (67.1\%) [8], and Asella Teaching Hospital (79.8\%) [10]. The treatment success in Arsi-Robe Hospital showed an increment during the first two years (76.6\% in 2013 and $86.8 \%$ in 2014) followed by an inconsistent increase and decrease in the following years (Table 4). The national treatment success rate (NTSR) was $91 \%$ in 2013 [41], 89\% in 2014 [6], 84\% in 2015 [42] and 90\% in 2016 [1]. This shows that the treatment success of Arsi-Robe Hospital was lower than the NTSR except in 2016 (90\%) (Table 4).

In multivariable logistic regression, the odd of unsuccessful treatment outcome of TB was higher in TB patients residing urban areas than rural residents (Table 4). It was agreement with recent study in Debre Tabor, Northern Ethiopia [23] and Sidama Zone, Ethiopia [15], but against study in south Ethiopia [13]. A model based study from India indicated that average treatment success rate decrease with increase in the proportion of urban population and with increase higher degrees of urbanization [43]. Comorbidities (HIV, and diabetes mellitus), temporary interruption of treatment and other addiction related factors were suggested for poor treatment outcome of TB among urban residents [23], and difficulties in ensuring quality of care and prevention of infection and transmission in densely populated areas with large disparities in sanitation and access to health care [43] have been suggested to explain the observed difference.

TB patients with failure treatment had high odd of significant unsuccessful treatment as compared to new patients (the reference patient category). This was in agreement with the study of Melesse and Zeleke (2018) [23] where new cases have lower odd poor treatment as compared to retreatment cases. It was found that previously treated TB cases (including failure) had a multidrug resistance TB (MDR) $[11,44]$ and was associated with unsuccessful treatment outcomes $[10,44]$.

In our study, TB patients with HIV co-infection had higher odd of unsuccessful treatment of TB as compared HIV negative TB patients. Several studies documented lower TSR of the HIV/TB co-infected cases [9-10, 13]. HIV co-infection also increases the risk of latent TB reactivation 20-fold, is the most known risk factor for progression of $M$. tuberculosis infection to active disease $[18,33]$. The low treatment success in TB/HIV co-infected patients could be due to co-administration of ART along with anti-TB therapy which can lead to drug-drug interactions, overlapping drug toxicities and immune reconstitution syndrome [45].

Smear positive pulmonary TB patients ( $\mathrm{PTB}+$ ) had lesser odd of unsuccessful treatment of TB as compared to smear negative pulmonary TB and extra pulmonary TB patients. This is in agreement with studies in Ethiopian University Hospital [9], and Southern Ethiopia [13]. According to Biruk et al. [9], there is a monitoring of the treatment outcome and testing of the sputum in the 2nd, 5th and 7th months of treatment for PTB + but for PTB- and EPTB, monitoring is only in clinical conditions. HIV coinfection in PTB- and EPTB patients may decrease the treatment success and increase mortality [6]. Global reports also indicated an increase in TB treatment success from 2012 to 2016 [1, 42]. The higher TSR of PTB + in our study could be attributed to improved diagnosis and good adherence to DOTs program in the Arsi-Robe Hospital.

This study was performed on retrospective basis and used already registered socio-economic and clinical data. As compared to WHO international target (85\%) $[11,18]$, our study revealed an appreciable treatment success rate of TB (84\%) in the study area. 
book of the hospital. Secondly, this study doesn't find data on other potential factors such as sputum smear test result at second month after initiation treatment, duration, malnutrition status, drug resistance pattern of the TB patients. The data was also collected from a single hospital, though it gives treatment services to more than a half a million of people of the town and the surrounding district. The absence of these critical factors has impact on the conclusion of the results of our study and any interpretation of the results should consider the mentioned limitations, and based on the variables considered.

\section{Conclusions}

The overall TB treatment success rate for all registered patients was $84.0 \%$ which comprised of $31.5 \%$ of cure and $52.5 \%$ of completion of treatment. The unsuccessful treatment outcome rate was $16 \%$ constituted by $5.8 \%$ of death, $5.1 \%$ default and $5.1 \%$ treatment failure. In multivariate analysis, residence of the patients, TB patients' category, HIV status of the patients and TB types were the predictor variables for unfavorable treatment success of tuberculosis in Arsi-Robe Hospital, Ethiopia. TB/HIV coinfected patients were found to have lesser chance of successful treatment success. It is therefore, important to strengthen the antiretroviral treatment of HIV coverage in order to reduce the morbidity and mortality due to TB. The higher proportion of PTBover and PTB + and EPTB in our study was also unusual as compared to many studies; therefore diagnosis of TB types should be confirmed by rapid and reliable methods with higher sensitivity and specificity. It is also essential to enhance strategy of TB patients' follow up and tracing to keep them in contact with health professionals at service delivery point during treatment could increase the successfulness of TB treatment outcome. It is also important to study the relation of other socio-economic factors and clinical factors with treatment outcome in the study area in other studies.

\section{Abbreviations}

AOR: Adjusted odds ratio; COR: Crude odds ratio; DOTs: Direct observed treatment strategies; EPTB: Extrapulmonary tuberculosis; HIV: Human immune deficiency virus; MDR-TB: Multidrug-resistant tuberculosis; NTLCP: National Tuberculosis and Leprosy Control Program; PTB+: Smear positive pulmonary tuberculosis; PTB-: Smear negative pulmonary tuberculosis; SPSS: Statistical package for social science; TB: Tuberculosis; VIF: Variance Inflation Factor (VIF); WHO: World Health Organization

\section{Declarations}

\section{Acknowledgments}

We are very much thankful for the staff of Arsi-Robe Hospital for their cooperation during our data collection process. This research was financially supported by Madda Walabu University. The authors did not have conflict of interest.

\section{Authors' Contributions}

AG carried out conceptualization of the research, visualize and validate the study design, performed the statistical analysis; and write, both the original draft and final manuscript. HK participated in the validation and visualization of the study design and statistical analysis and final editing of the manuscript. AA participated in the conceptualization of the study design, visualize and validate the study design, reviewed both the original draft and the final manuscript. All authors read and approved the final manuscript.

\section{Funding}

Not applicable.

\section{Availability of data and materials}

All the data and materials used in this paper will be available upon request.

\section{Ethics approval and consent to participate}


The study design and procedure of this research was approved by the Departmental Graduate council of the Department of Biology of Madda Walabu University. Ethical clearance was obtained from Madda Walabu University, College of Natural and Computational Sciences. Permission was sought from the Arsi-Robe Hospital administration before data collection. Patient records was anonymized and de-identified prior to analysis to ensure confidentiality of individual patient information.

\section{Consent for publication}

Not applicable.

\section{Competing interests}

The authors declare that they have no competing interests

\section{Authors' Details}

${ }^{1}$ Department of Biology, College of Natural and Computational Science, Madda Walabu University, Bale Robe, Ethiopia;

${ }^{2}$ Department of Epidemiology and Biostatistics, University of California, San Francisco, USA; email: helmutk@comcast.net

\section{References}

1. World Health Organization. Global Tuberculosis Report 2018 Document WHO/CDS/TB/2018.20; Geneva. 2018. Available from: https://apps.who.int/iris/bitstream/handle/10665/274453/9789241565646-eng.pdf.

2. World Health Organization. Tuberculosis Fact Sheet No. 104, 2010; Geneva: 2010. Available from: http://www.who.int/mediacentre/factsheets/fs104/en/print.html.

3. World Health Organization. Global Tuberculosis Report 2013 Document WHO/HTM/TB/2013.11. Geneva: 2013. Available from: https://apps.who.int/iris/bitstream/handle/10665/91355/9789241564656_eng.pdf.

4. Castelnuovo B. A review of compliance to antituberculosis treatment and risk factors for defaulting treatment in Sub Saharan Africa. Afr Health Sci. 2010;10(4):320-4.

5. Duarte R, Lönnroth K, Carvalho C, Lima F, Carvalho ACC, Muňoz-Torrico M, Centis R. Tuberculosis, social determinants and co-morbidities (including HIV). Pulmonol. 2018;24(2):115-9.

6. World Health Organization. Global Tuberculosis Report 2016 Document WHO/HTM/ TB/2016.13; Geneva: 2016. Available from: https://apps.who.int/iris/bitstream/handle/10665/250441/9789241565394-eng.pdf.

7. Ministry of Health of Ethiopia. Tuberculosis, Leprosy, Prevention TB/HIV and Control Programme Manual. 4th ed. Ministry of Health of Ethiopia (MOH): Addis Ababa, Ethiopia; 2008.

8. Tachbele E, Taye B, Tulu B, Ameni G. Treatment outcomes of tuberculosis patients at Bale Robe Hospital Oromia Regional State, Ethiopia: A five year retrospective study. J Nurs Care. 2017;6(2):386.

9. Biruk M, Yimam B, Abrha H, Biruk S, Amdie FZ. Treatment outcomes of tuberculosis and associated factors in an Ethiopian University hospital. Adv Public Health. 2016;2016:1-9 Available from: http://www.hindawi.com/ journals/aph/2016/8504629/.

10. Tafess K, Beyen TK, Abera A, Tasew G, Mekit S, Sisay S. Tadesse L, Siu GKH. (2018).Treatment outcomes of tuberculosis at Asella teaching hospital, Ethiopia: Ten years' retrospective aggregated data. Front Med. 2018;5:38.

https://doi.org/10.3389/fmed.2018.00038.

11. World Health Organization. Global Tuberculosis Report 2012 Document WHO/HTM/TB/2012; Geneva: 2012. Available from: https://www.who.int/tb/publications/global_report/gtbr12_main.pdf.

12. Federal Ministry of Health of Ethiopia. Guidelines for Clinical and Programmatic Management of TB, TB/HIV and Leprosy. 5th ed. Federal Ministry of Health of Ethiopia (FMOH): Addis Ababa, Ethiopia; 2012. 
13. Gebrezgabiher G, Romha G, Ejeta E, Asebe G, Zemene E, Ameni G. Treatment outcome of tuberculosis patients under directly observed treatment short course and factors affecting outcome in Southern Ethiopia: A five-year retrospective study. PLoS One. 2016;11(2):e0150560.

14. Hamusse SD, Demissie M, Teshome D, Lindtjørn B. Fifteen-year trend in treatment outcomes among patients with pulmonary smear-positive tuberculosis and its determinants in Arsi Zone, Central Ethiopia. Glob Health Action. 2014;7:25382. http://dx.doi.org/10.3402/gha.v7.25382.

15. Dangisso MH, Gemechu DG, Lindtjørn B. Trends of tuberculosis case notification and treatment outcomes in the Sidama Zone, Southern Ethiopia: Ten-year retrospective trend analysis in urban-rural settings. PLoS ONE. 2014;9(12):e114225.

16. Melese A, Zeleke B, Ewnete B. Treatment outcome and associated factors among tuberculosis patients in Debre Tabor, Northwestern Ethiopia: A retrospective study. Tuber Res Treat. 2016; 2016:1354356. http://dx.doi.org/10.1155/2016/1354356.

17. Wen Y, Zhang Z, Li X, Xia D, Ma J, Dong Y, Zhang X. Treatment outcomes and factors affecting unsuccessful outcome among new pulmonary smear positive and negative tuberculosis patients in Anqing, China: a retrospective study. BMC Infect Dis. 2018;18(1):104. https://doi.org/10.1186/s12879-018-3019-7.

18. World Health Organization. Global Tuberculosis Report 2014 Document WHO/HTM/TB/2014.08; Geneva: 2014. Available from: https://apps.who.int/iris/bitstream/handle/10665/137094/9789241564809_eng.pdf.

19. Narasimhan P, Wood J, Macintyre CR, Mathai D. Risk factors for tuberculosis. Pulmon Med. 2013;2013:828939. http://dx.doi.org/10.1155/2013/828939.

20. Mohammed MG, Norman ZN, Yoswa MD. Factors influencing treatment outcomes in tuberculosis patients in Limpopo Province, South Africa, from 2006 to 2010: a retrospective study. Curationis. 2014;37(1):7. http://dx.doi.org/10.4102/curationis.v37i1.1169.

21. Neyrolles O, Quintana-Murci L. Sexual inequality in tuberculosis. PLoS Med. 2009;6(12):e1000199.

22. World Health Organization. Global Tuberculosis Report 2019 Document WHO/CDS/TB/2019.15;Geneva: 2019. https://apps.who.int/iris/bitstream/handle/10665/329368/9789241565714-eng.pdf.

23. Melese A, Zeleke B. Factors associated with poor treatment outcome of tuberculosis in Debre Tabor, northwest Ethiopia. BMC Res Notes. 2018;11:25. https://doi.org/10.1186/s13104-018-3129-8.

24. Campos LC, Rocha MVV, Willers DMC, Silva DR. Characteristics of patients with smear-negative pulmonary tuberculosis (TB) in a region with high TB and HIV prevalence. PLoS ONE. 2016;11(1):e0147933. https://doi.org/10.1371/journal.pone.0147933.

25. Mekonnen D, Derbie A, Desalegn E. TB/HIV co-infections and associated factors among patients on directly observed treatment short course in Northeastern Ethiopia: a 4 years retrospective study BMC Res Notes. 2015. 8:666. https://doi.org/10.1186/s13104-015-1664-0.

26. Ali SA, Mavundla TR, Fantu R, Awoke T. Outcomes of TB treatment in HIV co-infected TB patients in Ethiopia: a crosssectional analytic study. BMC Infect Dis. 2016;16:640. https://doi.org/10.1186/s12879-016-1967-3.

27. Nyamogoba DN, Mbuthia G, Mining S, Kikuvi G, Biegon R, Mpoke S, et al. HIV co-infection with tuberculosis and nontuberculous mycobacteria in western Kenya: challenges in the diagnosis and management. Afr Health Sci. 2012;12(suppl 3):305-1128.

28. Datiko DG, Yassin MA, Chekol LT, Kabeto LE, Lindtjorn B. The rate of TB-HIV co-infection depends on the prevalence of HIV infection in the community. BMC Public Health. 2008;8:266. https://doi.org/10.1186/1471-2458-8-266.

29. Corbett EL, Watt CJ, Walker N, Maher D, Williams BG, et al. The growing burden of tuberculosis: global trends and interactions with the HIV epidemic. Arch Intern Med. 2003;163:1009-21.

30. Foulds J, O'Brien R. New tools for the diagnosis of tuberculosis: the perspective of developing countries. Int J Tuberc Lung Dis. 1998;2:778-83.

31. Sebhatu A. The Implementation of Ethiopia's Health Extension Program: An Overview, Addis Ababa, Ethiopia. 2008. https://www.phe-ethiopia.org/pdf/Health\%20Extension\%20Program\%20in\%20Ethiopia.pdf.

Loading [MathJax]/jax/output/CommonHTML/fonts/TeX/fontdata.js

Page 18/19 
32. World Health Organization. Global tuberculosis control 2009, Epidemiology, Strategy, Financing, Document. WHO/HTM/TB/2009.411;Geneva:2009.https://apps.who.int/iris/bitstream/handle/10665/44035/9789241563802_eng.pdf.

33. Getahun B, Ameni G, Medhin G, Biadgilign S. Treatment outcome of tuberculosis patients under directly observed treatment in Addis Ababa. Ethiopia. 2013;17(5):521-8. http://dx.doi.org/10.1016/j.bjid.2012.12.010.

34. Sileshi B, Deyessa N, Girma B, Melese M, Suarez P. Predictors of mortality among TB-HIV Co-infected patients being treated for tuberculosis in Northwest Ethiopia: A retrospective cohort study. BMC Infect Dis. 2013;1(13):297. https://doi.org/10.1186/1471-2334-13-297.

35. Cayla J, Caminero J, Rey R, Lara N, Valles X, Galdos-Tanguis H. Current status of treatment completion and fatality among tuberculosis patients in Spain. Int J Tuberc Lung Dis. 2004;8(4):458-64. https://doi.org/10.1371/journal.pone.020546.

36. Tesema T, Seyoum D, Ejeta E, Tsegaye R. Determinants of tuberculosis treatment outcome under directly observed treatment short courses in Adama City, Ethiopia. PLoS ONE. 2020;15(4):e0232468.

37. Sinshaw Y, Alemu S, Fekadu A, Gizachew M. Successful TB treatment outcome and its associated factors among TB/HIV co-infected patients attending Gondar University Referral Hospital, Northwest Ethiopia: an institution based cross-sectional study. BMC Infect Dis. 17:132. https://doi.org/10.1186/s12879-017-2238-7.

38. Tola A, Mishore KM, Ayele Y, Mekuria AN, Legese N. Treatment outcome of tuberculosis and associated factors among TBHIV co-infected patients at public Hospitals of Harar town, Eastern Ethiopia: A five year retrospective study. BMC Public Health. 2019;19:1658. https://doi.org/10.1186/s12889-019-7980-x.

39. Ejeta E, Beyene G, Balay G, Bonsa Z, Abebe G. Factors associated with unsuccessful treatment outcome in tuberculosis patients among refugees and their surrounding communities in Gambella Regional State, Ethiopia. PLoS ONE. 2018;13(10). https://doi.org/10.1371/journal.pone.0205468.

40. Mibei D, Kiarie J, Wairia A, Kamene M, Okumu M. Treatment outcomes of drug-resistant tuberculosis patients in Kenya. Int J Tuberc Lung Dis. 2016;20(11):1477-82.

41. World Health Organization. Global Tuberculosis Report 2015 Document WHO/HTM/TB/2015.22; Geneva: 2015. Available from: https://apps.who.int/iris/bitstream/handle/10665/191102/9789241565059_eng.pdf.

42. World Health Organization. Global Tuberculosis Report 2017 Document WHO/HTM/TB/2017.23; Geneva:2017. Available from: http://www.who.int/tb/publications/global_report/gtbr2017_main_text.pdf.

43. Singh $\mathrm{H}$, Ramamohan V. A model based investigation into urban-rural disparities in tuberculosis treatment outcomesunder the Revised National Tuberculosis Control Programme in India. PLoS ONE. 2020;15(2):e0228712.

44. Assefa D, Seyoum B, Oljira L. Determinants of multidrug-resistant tuberculosis in Addis Ababa, Ethiopia. Infect Drug Resist. 2017;10:209-13.

45. Padmapriyadarsini C, Narendran G, Swaminathan S. Diagnosis and treatment of tuberculosis in HIV co-infected patients. Indian J Med Res. 2011;134(6):850-65. 